\title{
Endo-Illumination-Aided Scleral Buckling Combined with Intravitreal Injection of Hyaluronate for Treatment of Rhegmatogenous Retinal Detachment
}

\author{
Tong Zhao Yujie Yan You Chen Chuan Sun Zhijun Wang \\ Department of Ophthalmology, China-Japan Friendship Hospital, Beijing, China
}

\section{Keywords}

Rhegmatogenous retinal detachment - Scleral buckling .

Noncontact wide-angle viewing system .

Endo-illumination · Intravitreal injection of hyaluronate

\begin{abstract}
Purpose: To evaluate the efficacy and safety of 25-gauge illumination-aided scleral buckling (SB) combined with hyaluronate injection for the treatment of rhegmatogenous retinal detachment (RRD). Methods: Forty-five cases were included in this retrospective study. Twenty-five cases of the treatment group received SB with the aid of endo-illumination and noncontact wide-angle viewing system combined with hyaluronate injection after subretinal fluid drainage, while 20 cases of the control group received conventional SB with binocular indirect ophthalmoscope combined with air injection. Best-corrected visual acuity (BCVA), intraocular pressure (IOP), and complications were observed and recorded. Results: The mean operation duration of the treatment group (42.06 $\pm 16.77 \mathrm{~min})$ was significantly shorter than that of the control group $(50.19 \pm 21.61 \mathrm{~min}, p=0.042)$. Only 1 case of the control group underwent a second surgery, and the final reattachment ratios of the 2 groups were both $100 \%$. BCVA improved in both the treatment group (from $0.91 \pm 0.79$ to $0.42 \pm 0.58 \log M A R, p<0.001$ ) and the
\end{abstract}

control group (from $0.82 \pm 0.70$ to $0.41 \pm 0.37 \log \mathrm{MAR}, p=$ 0.001). The improvements of BCVA of the treatment group and control group were $-0.49 \pm 0.38$ and $-0.42 \pm 0.46$ logMAR, with no significant difference $(p=0.594)$. There was no significant difference in IOP and complications between the 2 groups. Conclusions: 25-gauge endo-illumination-aided SB combined with hyaluronate injection was safe and effective for RRD.

(c) 2020 S. Karger AG, Basel

\section{Introduction}

Primary rhegmatogenous retinal detachment (RRD) is the most common type of retinal detachment and is an acute vision-threatening disease in ophthalmology [1]. Scleral buckling (SB) had been the leading approach since introduced by Schepens et al. [2] in the 1950s until the 1980 s with the advancement of pars plana vitrectomy [3]. A successful repair surgery relies mainly on the accurate scleral location of the retinal breaks [4]. However, inverted images observed by indirect ophthalmoscope and low magnification [5] bring difficulties for less experienced surgeons, which may lead to the omission of minimal retinal lesions resulting in unsuccessful operation [6]. Endoillumination with a wide-angle viewing system has been karger@karger.com

(c) 2020 S. Karger AG, Basel

www.karger.com/oph

Karger"
Zhijun Wang

Department of Ophthalmology

China-Japan Friendship Hospital

2 Yinghua East Road, Chaoyang District, Beijing 100029 (China)

E-Mail wangzhijuncj@sina.com 
introduced as a successful alternative method for retina visualization in SB [7]. Another concerned question is air injection after subretinal fluid (SRF) drainage. The air bulb may influence observation and easily escape from vitreous cavity [8]. To overcome the drawbacks of air injection, we use hyaluronate to maintain intraocular pressure (IOP) instead of air and observe the safety and efficacy of SB with endo-illumination and intravitreal injection of hyaluronate.

\section{Methods}

This was a retrospective, non-randomized controlled trial. This study was conducted based on the approval of the Ethics Committee of China-Japan Friendship Hospital and in accordance with the tenets of the Declaration of Helsinki. All patients understood the risks, benefits, and alternatives of the surgeries and signed informed consent. From January 2015 to December 2017, forty-five eyes of 45 patients of RRD who needed SB surgery were enrolled in the study. Exclusion criteria were as follows: (1) recurrent retinal detachment, (2) vitrectomized eyes, (3) macular hole retinal detachment or retinal holes of posterior pole, (4) proliferative vitreoretinopathy grade C2 [9] or more severe, (5) large retinal tear ranging $>3$ clock hours, and (6) obvious opacity of cornea or/and lens. Essential information of all patients was recorded: age, gender, time of onset, ocular and systemic disease history, best-corrected visual acuity (BCVA), IOP, lens status, number of retina breaks, location of retinal breaks, number of quadrants involved, the presence or absence of macular detachment. Axial length was measured with immersion A-scan ultrasound biometry. Patients were assigned into 2 groups according to different procedures: the treatment group (28 eyes) received endo-illumination-aided SB surgery, while the control group (20 eyes) received conventional SB with a binocular indirect ophthalmoscope.

\section{Endo-Illumination-Aided SB}

Conjunctiva peritomy was performed $180-360^{\circ}$ according to the site of the retinal break and the range of RRD, and extraocular muscle was isolated. A 25-G valved trocar cannula (Alcon Surgical Inc.) was placed $4.0 \mathrm{~mm}$ behind the limbus, keeping away from the location of retinal breaks. A noncontact wide-angle viewing system (Resight 700; Carl Zeiss Meditec AG) was activated. A fiber optic illuminator (Alcon Surgical Inc., Fort Worth, TX, USA) was inserted through the valved trocar. A full examination of the fundus was performed to locate all the retinal breaks and detect all $360^{\circ}$ peripheral areas to prevent omission of minimal lesions. Cryopexy was performed if there was no significant fluid near breaks. Otherwise, SRF drainage through the external sclerotomy was performed under the operating microscope at some distance away from retinal breaks to minimize the passage of vitreous through the breaks. Viscoelastic solution (Pe-Ha-Luron ${ }^{\circledR} \mathrm{F} 1.8 \%$, Albomed $\mathrm{GmbH}$ ) was injected into the vitreous cavity through the trocar to maintain IOP stable (Fig. 1a). The segmental buckling procedure was performed using silicone sponge after marking the retinal breaks on the sclera. A circumferential SB was performed in cases of multiple breaks or wide-range degeneration areas. Fiber illuminator was inserted once again to confirm the buckle position

Use of Endo-Illumination and

Hyaluronate in RRD and re-examine fundus. Cannula was removed, and conjunctiva was closed with an $8-0$ absorbable suture.

\section{Conventional SB}

Binocular indirect ophthalmoscope was used to examine fundus, locate retinal breaks, and perform cryopexy. The same drainage techniques were implemented when necessary while disinfected air was injected into vitreous cavity to keep a stable IOP. After routine buckling, fundus was checked using binocular indirect ophthalmoscope. Conjunctiva peritomy and suture were the same as described above.

Operation details were also recorded, including operation duration, whether performing external SRF drainage, and whether performing circumferential SB. Routine examinations were performed at 1,3, and 7 days and 1, 3, and 6 months postoperatively, including BCVA, IOP, and fundoscopy. Complications were also monitored and recorded, such as vitreous hemorrhage, subretinal hemorrhage, endophthalmitis, iatrogenic retinal breaks, and so on. A complete absorption of SRF in 7 days after the surgery was considered primary reattachment. A second surgery of SB or vitrectomy was performed if retina was not attached after the first surgery or retinal detachment recurred. The final reattachment ratio was calculated at 6 months after the primary surgery.

Decimal value of visual acuity was converted into the logarithm of the minimum angle of resolution $(\log M A R)$ for statistical analysis. Statistical analyses were performed with SPSS version 22.0 (IBM Corp., Armonk, NY, USA); Independent sample $t$ test, chisquare test, or Fisher exact test were used to analyze intergroup differences. Paired samples $t$ tests were used to compare preoperative and postoperative data.

\section{Results}

\section{Demographic Information and Baseline}

The mean age of the 45 patients was $34.9 \pm 12.5$ (range $20-67)$ and 18 of them (40\%) were female. The treatment group had a mean age of $36.2 \pm 11.7$ years and 9 females (36\%), while in the control group, there were 9 females $(45 \%)$, and the mean age was $33.5 \pm 10.4$ years. The differences in gender or age between the 2 groups were not statistically significant ( $p=0.572$ and $p=0.398)$. The BCVA of the treatment group and control group were $0.91 \pm 0.79$ and $0.82 \pm 0.70 \log M A R$, respectively, with no significant difference $(p=0.704)$. The IOP of the 2 groups were $13.17 \pm 3.11$ and $12.88 \pm 3.05 \mathrm{~mm} \mathrm{Hg}$, respectively $(p=0.753)$. The mean axial length of the treatment group was $25.91 \pm 2.33 \mathrm{~mm}$, with no significant difference compared with the control group $(25.55 \pm 4.71 \mathrm{~mm}, p=0.436)$. Fifteen patients were myopic in the treatment group, while the number of the control group was $14(p=0.486)$. None of the 2 groups had a history of ocular trauma.

Two eyes of the treatment group and one of the control group were pseudophakia $(p=0.585)$. One pseudophakic eye of the treatment group had a contracted anterior cap- 
Fig. 1. a Hyaluronate was injected into vitreous cavity through the trocar after drainage of SRF. Arrow shows gelatinous hyaluronate. b Clear fundus view after hyaluronate was injected. Arrow shows hyaluronate in vitreous cavity.
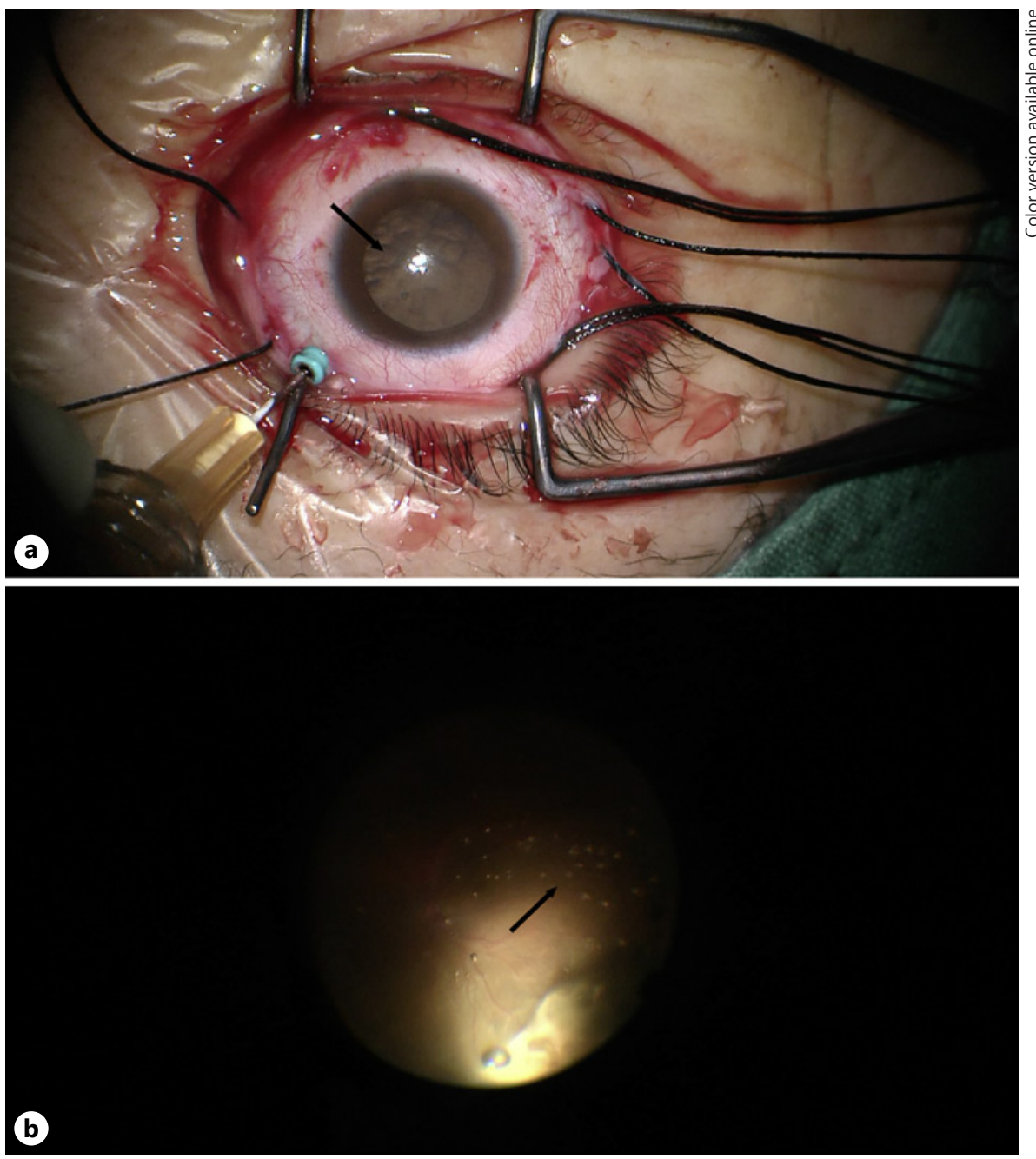

sule and a small anterior capsular opening of $4 \mathrm{~mm}$, which provided a poor view of the fundus; 6-clock-hour detachment with a retinal fold in the inferotemporal quadrant could be seen, but the confirmed tear was not found before the surgery. There were no statistical differences as for the comparison of location of breaks ( $p=0.589$ ), cases of macula off $(p=0.731)$, clock hours of detachment extension $(p=0.840)$, and proliferative vitreoretinopathy grade $(p=0.895)$. Details are shown in Table 1 .

\section{The Surgeries}

The mean operation duration of the treatment group was $42.06 \pm 16.77 \mathrm{~min}$, significantly shorter than the control group (50.19 $\pm 21.61 \mathrm{~min}, p=0.042)$. In the treatment group, one circle hole was found during the operation of the pseudophakic eye mentioned above, and in another case, one additional hole that had not been detected pre- operatively was found aided by endo-illumination. No additional hole was detected in the control group. In the treatment group, local buckling was done in all 25 eyes, additional encircling band was used in 2 eyes, and SRF drainage combined with hyaluronate injection was performed in 12 eyes. In the control group, local buckling was done in all 20 eyes, additional encircling band was used in 3 eyes, and SRF drainage combined with gas injection was conducted in 10 eyes.

Subretinal hemorrhage with a one-disc-diameter area occurred in one case when performing drainage through external sclerotomy in the treatment group. Trace vitreous hemorrhage was observed in one case of the control group during the surgery. The hemorrhages of the 2 cases were completely resolved within 7 days after the surgery. No lens injury, iatrogenic retinal tear, or acute suprachoroidal hemorrhage was recorded in both groups. 
Table 1. Demographics and baseline

\begin{tabular}{lccc}
\hline & Treatment group & Control group & $p$ value \\
\hline Number of patients/eyes & $25 / 25$ & $20 / 20$ & $0.715^{*}$ \\
Age, years (range) & $36.2 \pm 11.7(20-67)$ & $33.5 \pm 10.4(24-58)$ & $0.540^{* *}$ \\
Gender, female & $9(36)$ & $9(45)$ & $0.704^{*}$ \\
BCVA, logMAR & $0.91 \pm 0.79$ & $0.82 \pm 0.70$ & $0.753^{*}$ \\
IOP, mm Hg & $13.17 \pm 3.11$ & $12.88 \pm 3.05$ & $0.436^{*}$ \\
Axial length, mm & $25.91 \pm 2.33$ & $25.55 \pm 4.71$ & - \\
History of ocular trauma & $0(0)$ & $0(0)$ & $0.486^{* *}$ \\
Myopia & $15(60)$ & $14(70)$ & $0.585^{* * *}$ \\
IOL & $2(8)$ & $1(5)$ & $0.598^{* * *}$ \\
Number of breaks & 35 & 28 & $0.731^{* *}$ \\
Location of the breaks & & & $0.840^{*}$ \\
$\quad$ Pre-equatorial & 15 & 14 & $0.895^{* * *}$ \\
$\quad$ Equatorial & 19 & 0 & 13 \\
$\quad$ Post-equatorial & 1 & $7.3 \pm 3.9$ & \\
Macula-off cases, $\%$ & 15 & 10 & \\
Clock hours of detachment extension & $7.5 \pm 4.7$ & 9 & \\
PVR grade & 11 & 1 & \\
$\quad$ A & 13 & 1 & \\
B & & & \\
C1 & & & \\
\hline
\end{tabular}

Values presented as $n(\%)$ or mean $\pm \mathrm{SD} .{ }^{*}$ Independent sample $t$ test. ${ }^{* *}$ Chi-square test. ${ }^{* * *}$ Fisher exact test.

Table 2. Follow-up characteristics

\begin{tabular}{llcl}
\hline & Treatment group & Control group & $p$ value \\
\hline Primary reattachment & $25(100)$ & $19(95)$ & $0.444^{* *}$ \\
Final reattachment & $25(100)$ & $20(100)$ & - \\
BCVA, logMAR & $0.42 \pm 0.58$ & $0.41 \pm 0.37$ & $0.930^{*}$ \\
Change of BCVA, logMAR & $-0.49 \pm 0.38$ & $-0.42 \pm 0.46$ & $0.594^{*}$ \\
IOP, mm Hg & $14.01 \pm 2.64$ & $14.59 \pm 2.69$ & $0.470^{*}$ \\
Complications & & & $0.556^{* *}$ \\
$\quad$ Subretinal hemorrhage & 1 & 0 & $0.444^{* *}$ \\
$\quad$ Vitreous hemorrhage & 0 & 1 & - \\
$\quad$ Iatrogenic retinal tear & 0 & 0 & - \\
$\quad$ Lens injury & 0 & 0 & - \\
$\quad$ Suprachoroidal hemorrhage & 0 & 0 & 0 \\
\hline
\end{tabular}

Values presented as $n(\%)$ or mean \pm SD. ${ }^{*}$ Independent sample $t$ test. ${ }^{* *}$ Fisher exact test.

\section{Postoperation}

All patients of the treatment group achieved complete absorption of SRF within 1 week after surgeries. One eye of the control group presented with persistent SRF while retinal break was positioned on the center of the buckle crest. Subsequent vitrectomy combined with circle buckling and gas injection was performed, finding another omitted little hole. Retinal reattachment was achieved in all 45 eyes at the final follow-up (Table 2).

The mean follow-up period was $8.4 \pm 2.8$ months (616 months). At the final follow-up, both the treatment 


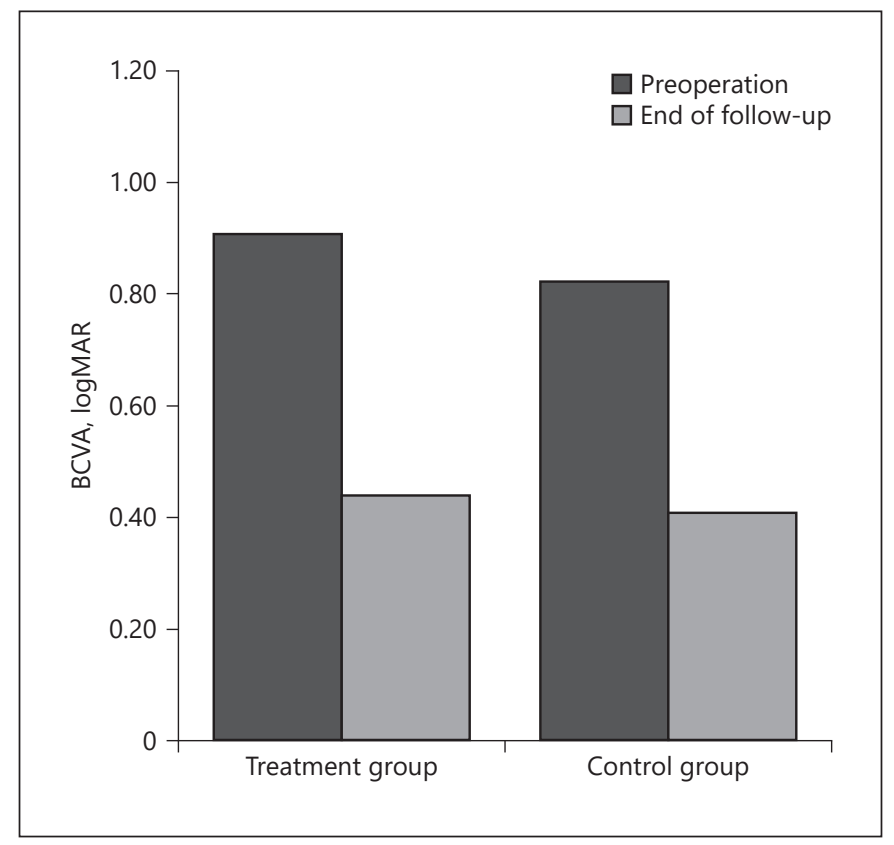

Fig. 2. The change of BCVA before the surgery and at the end of follow-up.

group and control group gained significantly improved BCVA $(0.42 \pm 0.58$ and $0.41 \pm 0.37 \log$ MAR, respectively) compared with preoperative BCVA (both $p<0.001$ ) (Table 3$)$. The changes of BCVA of the treatment group and control group were $-0.49 \pm 0.38$ and $-0.42 \pm 0.46$ logMAR, respectively. There were no significant differences when comparing the final BCVA $(p=0.930)$ or the change of BCVA ( $p=0.594$; Table 2; Fig. 2$)$.

Ten eyes of the treatment group and 7 of the control group underwent transient elevated IOP, which lowered to normal after using local and general glaucoma medications. Among the 17 eyes, 7 were hyaluronate injection cases, 5 were injected with air, and the remaining 5 had no injection, which showed no significant difference in different tamponades $(p=0.088)$. At the end of follow-up, the IOP of all patients from either group was no higher than $21 \mathrm{~mm} \mathrm{Hg}$ without using any glaucoma medication or undergoing glaucoma surgery; the mean IOP of the 2 groups were $14.01 \pm 2.64$ and $14.59 \pm 2.69 \mathrm{~mm} \mathrm{Hg}$, with no significant difference compared with preoperative values $(p=0.103$ and $p=0.085$, respectively; Table 3$)$. And no difference was found between the 2 groups (Table 2; Fig. 3).

No complications such as endophthalmitis, delayed suprachoroidal hemorrhage, recurrent retinal detachment, or cataract formation were observed (Table 2).

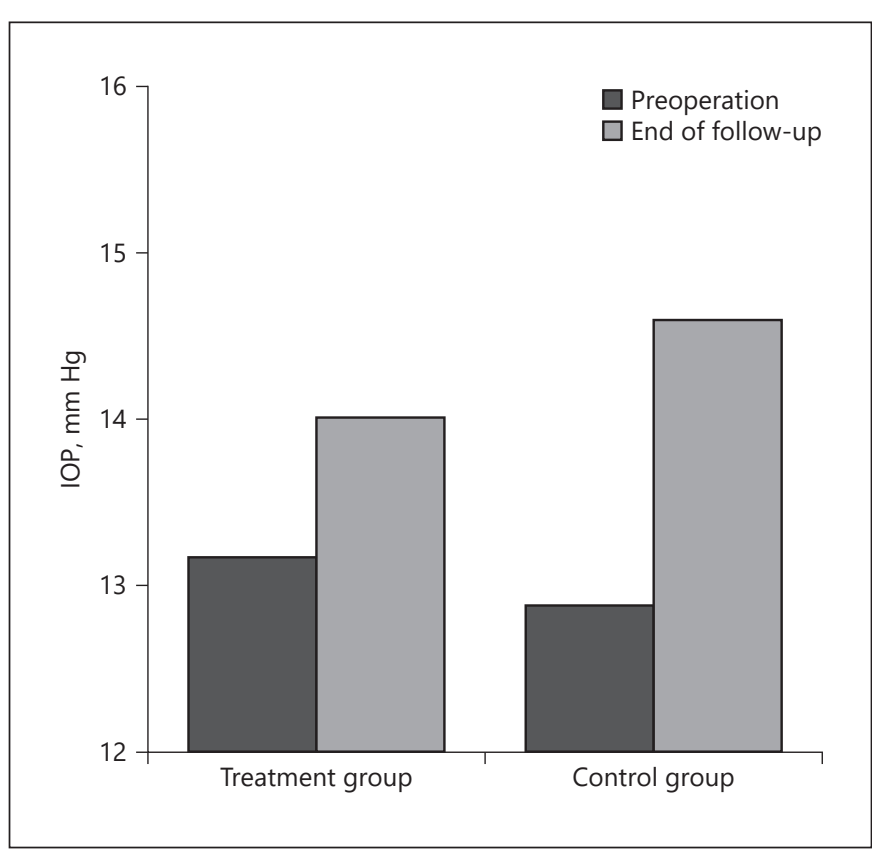

Fig. 3. The change of IOP before the surgery and at the end of follow-up.

Table 3. Change of BCVA (logMAR) and IOP (mm Hg)

\begin{tabular}{lccc}
\hline & Preoperation & $\begin{array}{l}\text { End of } \\
\text { follow-up }\end{array}$ & $p$ value \\
\hline BCVA & & & \\
$\quad$ Treatment group & $0.91 \pm 0.79$ & $0.42 \pm 0.58$ & $<0.001$ \\
$\quad$ Control group & $0.82 \pm 0.70$ & $0.41 \pm 0.37$ & $<0.001$ \\
IOP & & & \\
$\quad$ Treatment group & $13.17 \pm 3.11$ & $14.01 \pm 2.64$ & 0.103 \\
$\quad$ Control group & $12.88 \pm 3.05$ & $14.59 \pm 2.69$ & 0.085 \\
\hline
\end{tabular}

Paired $t$ test.

\section{Discussion}

From our study, it was found that endo-illuminationaided SB achieved a satisfactory result of reattachment ratio and BCVA improvement, same as conventional surgery using binocular indirect ophthalmoscope. However, we found the following advantages of the improved SB: (1) The use of endo-illumination and noncontact wideangle viewing system made a clearer view of fundus and it became easier to confirm the location of retinal tear. As mentioned above, one pseudophakic eye with a contracted and small-opening anterior capsule, making it hard to 
find retinal break preoperatively, underwent a successful SB with the aid of endo-illumination, which relieved the economic and physical stress of vitrectomy and silicone oil injection and freed patients from face-down position. In another case, one additional hole not detected preoperatively was found, which might avoid a recurrent retinal detachment. Furthermore, the only failed case of the control group was caused by an omitted hole. The better visualization of endo-illumination and noncontact wideangle viewing system could reduce recurrence rate by sealing all retinal holes, even those that were not identified preoperatively, and make SB an option for the cases with small pupils or opacity of refractive media [10]. (2) The improved procedure had a shorter mean operation duration than conventional surgery. Generally, it often took more time using an indirect ophthalmoscope to exam peripheral retina with scleral depression and move around patient instantly to obtain a 360-degree retinal view, which could be the explanation of the longer time. Therefore, the improved surgery would ease surgeons' stress caused by bending neck and waist repeatedly, to some extent. (3) Finally, surgical microscope enabled obtaining video records for further training and review.

Our study confirmed that using hyaluronate for intravitreal injection was safe and might be superior than air injection. Based on our previous study, it was found that proper drainage and buckling could guarantee complete closure of the retinal breaks at the end of the operation. Therefore, gas pressing against the hole using its surface tension was not necessary. The aim of injection after drainage could be limited in maintaining IOP. Hyaluronic acid would be a better choice. Although taken as a potential vitreous substitute for a long time [11], hyaluronic acid is now mainly used as the viscoelastic solution in cataract surgery. Intraocular use of hyaluronate, including intravitreal use, proved safe and nontoxic to retina $[12,13]$. The homogeneous refractivity of hyaluronate similar to vitreous maintained a clear visualization after intravitreal injection (Fig. 1b). On the contrary, the air bubble and even the "fish egg"-like bubbles in the vitreous after gas injection could greatly block the view of fundus [14]. As to pseudophakic eyes and those who had loose or damaged zonular suspension, gas might come into the anterior chamber, limiting retinal visualization dramatically. We observed 12 cases of hyaluronate injection following SRF drainage and found perfect fundus view. Temporarily elevated IOP was observed in 7 cases, which had no significant difference compared with gas injection or non-injection. No other complication was found. However, a potential disadvantage of hyaluronate injec- tion was that the formation of commuting flow between retinal tear and external sclerotomy hole might result in hyaluronate flowing out through the drainage hole or flowing to subretinal space. To avoid this situation, sclerotomy should be performed at a proper site far enough away from the retinal tear and the pinpoint of needle should not be oriented directly to the retinal tear when hyaluronate was injected. The phenomenon did not really occur in our 12 cases with careful operation and special attention to this point.

Despite zero occurrences in our study, the complications reported in previous literature should be considered, such as vitreous herniation from sclerotomy sites, vitreoretinal traction [6], endophthalmitis [15], iatrogenic retinal tears, or lens damage [5]. To reduce the complications, some points should be emphasized: (1) avoid inserting the illuminator probe into vitreous repeatedly, (2) avoid moving the probe rapidly and over-inserting, (3) do not stir vitreous, (4) examine the closure of sclerotomy site in case of vitreous herniation from sclerotomy sites and secondary vitreoretinal traction, (5) chandelier endoilluminators might be another choice for limited insertion depth and less possible stir $[10,16]$.

\section{Conclusions}

Both anatomical and functional results achieved by endo-illumination-aided SB were satisfactory, same as conventional SB. Intravitreal injection of hyaluronate instead of gas maintained a clear visualization and caused no extra complications. Endo-illumination-aided SB combined with hyaluronate injection was proven efficient and safe.

\section{Acknowledgement}

The author is grateful to Jingjing Jiang, MD, Zhike Zhang, MD, Yue Yin, MD, and Di Gong, MD at China-Japan Friendship Hospital.

\section{Statement of Ethics}

This study was conducted based on the approval of the Ethics Committee of China-Japan Friendship Hospital and in accordance with the tenets of the Declaration of Helsinki. All patients understood the risks, benefits, and alternatives of the surgeries and signed informed consent. Proved trial registration: ChiCTR1800020055. 


\section{Disclosure Statement}

The authors have no conflicts of interest to declare.

\section{Author Contributions}

Z.W.: study design and editing. T.Z.: drafting the manuscript, data analysis, literature search. Y.Y.: literature search. Y.C.: data analysis. C.S.: revision and editing.

\section{Funding Sources}

There are no founding sources to declare.

\section{References}

1 Poulsen CD, Peto T, Grauslund J, Green A. Epidemiologic characteristics of retinal detachment surgery at a specialized unit in Denmark. Acta Ophthalmol. 2016 Sep;94(6):54855.

2 Schepens CL, Okamura ID, Brockhurst RJ. The scleral buckling procedures. I. Surgical techniques and management. AMA Arch Opthalmol. 1957 Dec;58(6):797-811.

3 Haugstad M, Moosmayer S, Bragadóttir R. Primary rhegmatogenous retinal detachment - surgical methods and anatomical outcome. Acta Ophthalmol. 2017 May;95(3):247-51.

4 Noori J, Bilonick RA, Eller AW. Scleral buckle surgery for primary retinal detachment without posterior vitreous detachment. Retina. 2016 Nov;36(11):2066-71.

5 Aras C, Ucar D, Koytak A, Yetik H. Scleral buckling with a non-contact wide-angle viewing system. Ophthalmologica. 2012;227(2): $107-10$
6 Nam KY, Kim WJ, Jo YJ, Kim JY. Scleral buckling technique using a 25 -gauge chandelier endoilluminator. Retina. 2013 Apr;33(4): 880-2.

7 Yan H. Scleral buckling with a noncontact wide-angle viewing system in the management of rhegmatogenous retinal detachment. Eur J Ophthalmol. 2017 Jan;27(1):98-103.

8 Zhang Z, Peng M, Wei Y, Jiang X, Zhang S. Pars plana vitrectomy with partial tamponade of filtered air in Rhegmatogenous retinal detachment caused by superior retinal breaks. BMC Ophthalmol. 2017 May;17(1):64.

9 Hilton G, Machemer R, Michels R, Okun E, Schepens C, Schwartz A. The classification of retinal detachment with proliferative vitreoretinopathy. Ophthalmology. 1983 Feb;90(2): 121-5.

$10 \mathrm{Hu} \mathrm{Y,} \mathrm{Si} \mathrm{S,} \mathrm{Xu} \mathrm{K,} \mathrm{Chen} \mathrm{H,} \mathrm{Han} \mathrm{L,} \mathrm{Wang} \mathrm{X,} \mathrm{et}$ al. Outcomes of scleral buckling using chandelier endoillumination. Acta Ophthalmol. 2017 Sep;95(6):591-4.

11 Pruett RC, Schepens CL, Swann DA. Hyaluronic acid vitreous substitute. A six-year clinical evaluation. Arch Ophthalmol. 1979 Dec;97(12):2325-30.
12 Barth H, Crafoord S, Andréasson S, Ghosh F. A cross-linked hyaluronic acid hydrogel (Healaflow $\left({ }^{\circledR}\right)$ ) as a novel vitreous substitute. Graefes Arch Clin Exp Ophthalmol. 2016 Apr;254(4):697-703.

13 Kleinberg TT, Tzekov RT, Stein L, Ravi N, Kaushal S. Vitreous substitutes: a comprehensive review. Surv Ophthalmol. 2011 Jul-Aug; 56(4):300-23.

14 Caporossi T, Finocchio L, Barca F, Franco F, Tartaro R, Rizzo S. Scleral buckling for primary rhegmatogenous retinal detachment using a noncontact wide-angle viewing system with a cannula-based 27-G chandelier endoilluminator. Retina. 2019 Oct;39 Suppl 1:S144-50.

15 Sakono T, Otsuka H, Shiihara H, Yoshihara N, Sakamoto T. Acute bacterial endophthalmitis after scleral buckling surgery with chandelier endoillumination. Am J Ophthalmol Case Rep. 2017 Jul;8:7-10.

16 Seider MI, Nomides RE, Hahn P, Mruthyunjaya P, Mahmoud TH. Scleral Buckling with Chandelier Illumination. J Ophthalmic Vis Res. 2016 Jul-Sep;11(3):304-9. 